\title{
La escucha de música antes del TSST regula los niveles de cortisol en saliva independiente de la preferencia musical en estudiantes universitarios
}

\section{Listening to music before TSST modulates salivary cortisol levels in a nondependent way of music preference in college students}

DOI: 10.11144/Javeriana.upsy15-5.emrn

Recepción: 14 Julio 2016 | Aprobación: 28 Noviembre 2016

\author{
William Ruiz Gallo \\ Corporación Universitaria Minuto de Dios, Colombia \\ Óscar G. Rodríguez-Angarita \\ Corporación Universitaria Minuto de Dios, Colombia \\ Mayerli A. Prado-Rivera \\ Corporación Universitaria Minuto de Dios, Colombia \\ D. Carolina Cárdenas-Poveda ${ }^{\mathrm{a}}$ \\ Corporación Universitaria Minuto de Dios, Colombia
}

\footnotetext{
aCorrespondencia autor principal. Correo electrónico: dccardenasp@unal.edu.co

Para citar este artículo: Ruiz Gallo, W., Rodríguez-Angarita, O.G., Prado-Rivera, M. A., \& Cárdenas-Poveda, D. C. (2016). La escucha de música antes del TSST regula los niveles de cortisol en saliva independiente de la preferencia musical en estudiantes universitarios. Universitas Psychologica, 15(5). http:// dx.doi.org/10.11144/Javeriana.upsy15-5.emrn
}

\section{RESUMEN}

El presente estudio evaluó si la escucha de música preferida por los participantes o la música relajante elegida por los investigadores previa al protocolo de estrés social modificado TSST (Trier Social Stress Test) afectaba el desempeño de las actividades realizadas durante el propio test, modulaba la respuesta de estrés que este induce, y en conjunto con el estresor, afectaba el desempeño en una tarea de atención en setenta y seis estudiantes universitarios asignados a uno de seis grupos: música relajante del investigador, música preferida por el participante y silencio, con o sin TSST. Los resultados mostraron que la escucha de ambos tipos de música provocó una pendiente ascendente menos pronunciada en los niveles de cortisol en saliva luego del TSST, comparada con la que presentaron quienes no escucharon música, mientras que el aumento en la ansiedad-estado evaluada con el IDARE (Inventario de Ansiedad Rasgo-Estado) fue semejante en todos los grupos. No se encontraron diferencias debidas a la música en el desempeño de las tareas evaluadas. Se discute que la modulación de la escucha de música, independiente de la preferencia musical, tiene un efecto sobre la respuesta fisiológica de estrés posiblemente por la inducción de reacciones emocionales que esta provoca.

Palabras clave

cortisol en saliva; TSST; escucha de música relajante; preferencia musical; IDARE; salivary cortisol; TSST; listening to relaxing music; music preference; STAI.

\footnotetext{
ABSTRACT

The present study examines the effects of listening to music selected by participants or relaxing music chosen by researchers before modified TSST (Trier Social Stress Test) on: 1) TSST tasks, 2) TSST-induced stress responses, and 3) one attention task with both music and TSST before it. Seventy six college students were randomly assigned to one of
} 
William Ruiz Gallo, Óscar G. Rodríguez-Angarita, Mayerli A. Prado-Rivera, D. Carolina CárdenasPoveda.

six groups: listening to relaxing music chosen by researchers, previously selected music by students, or silence, any of them with or without TSST. Results showed that both relaxing or selected-by-participant music slowed salivary cortisol increase levels after TSST, whereas STAI (State-Trait Anxiety Inventory) increases were not significantly different. Listening to music has no significant effects on either TSST or attention tasks. Effects of listening to music on salivary cortisol levels due to possible emotional responses irrespective of music preference were discussed.

\section{Introducción}

La resolución de problemas que es frecuente en el desarrollo de actividades laborales y académicas, implica procesos como la atención y el aprendizaje. Diversos estudios en animales y humanos describen que estos procesos se relacionan con el funcionamiento del sistema neuroendocrino, en particular el efecto que ejerce el eje hipotálamo-pituitariosuprarrenal (HPA) y su principal producto, los glucocorticoides (GC), sobre la conducta (Lupien, Maheu, Tu, Fiocco, \& Schramek, 2007), sobre otros sistemas (i.e. cardiovascular, inmunitario), y sobre la capacidad de aprendizaje y memoria que se pueden ver comprometidas (Ortega, et al., 2013). Algunos estudios experimentales de los efectos de los GC sobre la corteza prefrontal o temporal en los que se altera la memoria de trabajo (Johnson, 2015; Lataster et al., 2011; Mizoguchi et al., 2000; Porcelli et al., 2008; Schoofs, Pabst, Brand, \& Wolf, 2013), o semántica (de Quervain et al., 2003), permiten suponer que la liberación de GC también puede afectar tareas fuera del laboratorio y que requieran procesos de atención, aprendizaje, o recobro de información, tales como algunas exigidas en contextos laborales o académicos.

El incremento en la liberación de GC por la activación del eje HPA en el sistema nervioso ocurre como parte de una cadena de recursos metabólicos que utiliza un organismo para afrontar una situación que lo pone en riesgo y que percibe como estresante (Schwabe et al., 2012). A partir de los estudios de Mason (1968) sobre las características psicológicas que podrían hacer estresante cualquier situación a la que se expusiera una persona, actualmente se describe que las situaciones novedosas, impredecibles, incontrolables y/o amenazantes para el yo pueden ser percibidas como estresantes y en consecuencia provocar la activación de los recursos en el sujeto para enfrentarla (Lupien et al., 2007). Usando como criterio la temporalidad de los eventos el estrés puede categorizarse en crónico o agudo. La exposición crónica tiene efectos negativos para la salud a mediano y largo plazo (McEwen, 2007), mientras que la exposición aguda tiene efectos que siguen un patrón de U-invertida sobre algunas tareas que exigen aprendizaje y memoria (Sandi, 2011): cuando el nivel de estrés es muy bajo o muy alto los efectos son deletéreos sobre la memoria declarativa pero son facilitadores cuando el nivel es medio (Het, Ramlow, \& Wolf, 2005; Lupien et al., 2007), sin embargo los efectos del estrés sobre la memoria de trabajo son contradictorios pues algunos estudios muestran que la exposición a estrés agudo puede mejorar el desempeño en este tipo de tareas cuando tienen una alta demanda cognitiva, (Duncko, Johnson, Merikangas, \& Grillon, 2009; Lai et al., 2014), mientras que otras investigaciones no evidencian efecto alguno (Porcelli et al., 2008) o indican que éste difiere según el género de los participantes (facilitador en los hombres y deletéreo en las mujeres, Schoofs et al., 2013)

Los protocolos controlados para inducir respuestas de estrés en humanos deben considerar la interpretación que el sujeto hace de la situación como una variable importante, ya que según como sea percibida, puede considerarse positiva y movilizar recursos para afrontarla (Lai et al., 2014). El Trier Social Stress Test (TSST) es el arreglo experimental mejor validado para inducir respuestas de estrés. Las personas que han sido evaluadas con este protocolo evidencian consistentemente un incremento significativo en la liberación de la hormona cortisol (Kudielka, Hellhammer, \& Kirschbaum, 2007), la cual se describe como el principal biomarcador de evaluación no invasiva comparado con la liberación de alfa-amilasa en saliva (sAA) o la tasa cardiaca, ya que estos 
son considerados inespecíficos y su incremento se evidencia en otras situaciones diferentes al estrés (Allen, Kennedy, Cryan, Dinan, \& Clarke, 2014). El protocolo original incluye dos tareas: una preparación y exposición oral como candidato para optar por un trabajo y el desarrollo de una tarea de cálculo aritmético, las cuales son evaluadas por supuestos expertos (Kirschbaum, Pirke, \& Hellhammer, 1993). Estudios con variaciones en este protocolo sugieren que sentirse evaluado es el principal factor que provoca estrés. Se han observado incrementos en los niveles de cortisol con modalidades de presentación distintas: presentación libre y cálculo aritmético (Schoofs \& Wolf, 2011); defensa ante una acusación de un supuesto robo y cálculo aritmético (Buchanan et al., 2010); presentación académica sobre un tema específico y cálculo aritmético (Tarazona, Cerón $\&$ Lamprea, 2013) y este aumento correlaciona con la valoración subjetiva de estrés en estudios que han usado escalas visuales analógicas o cuestionarios como el Inventario de Ansiedad Rasgo-Estado IDARE (Lai et al., 2014; Zwissler et al., 2011). De esta manera, el TSST ha evidenciado que las personas manifiestan respuestas de estrés cuando están expuestas ante otros que están emitiendo un juicio sobre ellos, y ha sido útil para evaluar lo que puede ocurrir en una situación real en contextos laborales y académicos.

Algunos estudios han evaluado factores que pueden reducir los niveles de cortisol y la activación del sistema inmunitario relacionados con situaciones de estrés. Por ejemplo, la escucha de música redujo significativamente los niveles de cortisol y de linfocitos en pacientes que se sometieron a cirugía (Leardi et al., 2007) y los niveles más bajos se reportaron en los pacientes que escucharon música preseleccionada por ellos (a partir de cuatro géneros propuestos: clásica, country, pop y dance) en comparación con los que escucharon música preseleccionada por los investigadores (new age). Además, la preferencia musical parece ser un factor adicional importante para la reducción de los niveles percibidos de dolor luego de una cirugía (Ebneshahidi \& Mohseni, 2008) y la percepción de estrés generada por la presentación de una tarea matemática (Jiang, Jun, Linshu, Daphne, \& Cunmei, 2013).

El efecto de la música sobre la percepción de estrés y sus indicadores fisiológicos puede deberse a su capacidad para influir en las emociones (Chanda \& Levitin, 2013): aquellas positivas están asociadas con incrementos en los niveles de dopamina (Ashby, Isen, \& Turken, 1999), y además parecen mejorar habilidades cognitivas como la toma de decisiones y la solución de problemas (Isen, 2009; Isen \& Labroo, 2003). Los estudios sobre el "efecto Mozart" han mostrado que habilidades visoespaciales pueden mejorar luego de la escucha de música compuesta por Mozart (Rauscher, Shaw, \& Ky, 1993). Aunque dicho efecto no siempre ha sido replicado, sistemáticamente se ha encontrado una mejora en el desempeño de diversos tipos de tareas en personas expuestas previamente a algún tipo de estimulación que les permita generar un estado emocional positivo i.e. escuchar la narración de una historia (Schellenberg, Glenn Schellenberg, $\&$ Weiss, 2013). Dicho efecto parece depender más del tipo de tarea, su dificultad (entre más difícil la tarea, mejor efecto de la escucha de música) y de los gustos particulares de los participantes (ver efecto Blur en Schellenberg \& Hallam, 2005), que del compositor de la pieza musical o del texto narrado.

Teniendo en cuenta lo anterior, el presente estudio tiene tres objetivos. El primero pretende identificar si la escucha de música con tempo lento, ritmo suave y sin letra seleccionada por los investigadores, denominada música relajante: (Knight \& Rickard, 2001; Khalfa, Bella, Roy, Peretz, \& Lupien, 2003; Nilsson, 2009) y/o preferida por el participante puede facilitar el desempeño de las actividades que se realizan durante el TSST. Estudios previos con este protocolo se han centrado en la respuesta de estrés inducida, pero no han evaluado la ejecución del cálculo aritmético ni el desempeño en la exposición oral, y la preferencia musical o las características relajantes de una pieza musical pueden modular respuestas emocionales que acompañan la respuesta de estrés. Este análisis puede indicar si el desempeño de tareas 
William Ruiz Gallo, Óscar G. Rodríguez-Angarita, Mayerli A. Prado-Rivera, D. Carolina CárdenasPoveda.

matemáticas y de exposición oral son afectados por la percepción de que la tarea puede ser estresante y si la escucha de música previa cambia tal percepción de estrés y facilita el desempeño. El segundo objetivo pretende evaluar los efectos de la escucha de música sobre la respuesta de estrés inducida por el TSST, ya que poco se han estudiado factores que puedan modular las respuestas de estrés inducidas por este protocolo. Este objetivo cobra importancia en la medida en que resulta una estrategia sencilla que permitiría afrontar en algunos casos eventos estresantes. El tercer objetivo busca identificar cuál es el desempeño en una tarea de atención sostenida y dividida en función de la música con las características descritas más el protocolo de estrés. En un estudio anterior, Tarazona et al. (2013) encontraron que el TSST no afecta el desempeño del Paced Auditory Serial Addition Test (PASAT), pero no se sabe si la escucha de música previa a la situación de estrés puede inducir una menor activación psicofisiológica, y en consecuencia modificar el desempeño en el PASAT.

\section{Método y materiales}

\section{Participantes}

Ochenta y tres estudiantes de psicología de la Corporación Universitaria Minuto de DiosSede principal (16 hombres y 67 mujeres), con edades entre 18 y 26 años $(X=20,91$, $s d=2,05)$ participaron voluntariamente en el estudio entre septiembre de 2014 y febrero de 2015. Se eliminaron del análisis final los resultados de cuatro participantes ya que la muestra de saliva obtenida fue insuficiente para detectar la concentración de cortisol, uno decidió retirarse antes de finalizar el protocolo, y dos se eliminaron porque los rangos de concentración de cortisol no correspondían con los valores de referencia, siendo 76 participantes la muestra final. En la tabla 1 se describen todos los grupos y las condiciones a las que estuvieron expuestos los participantes durante el protocolo. La asignación de cada uno a uno de los grupos fue hecha al azar.

\section{TABLA 1}

Abreviaturas de grupos con la condición del procedimiento para cada uno. A todos los participantes se les tomaron cuatro muestras de saliva cada 15 minutos para establecer los cambios en los niveles de cortisol.

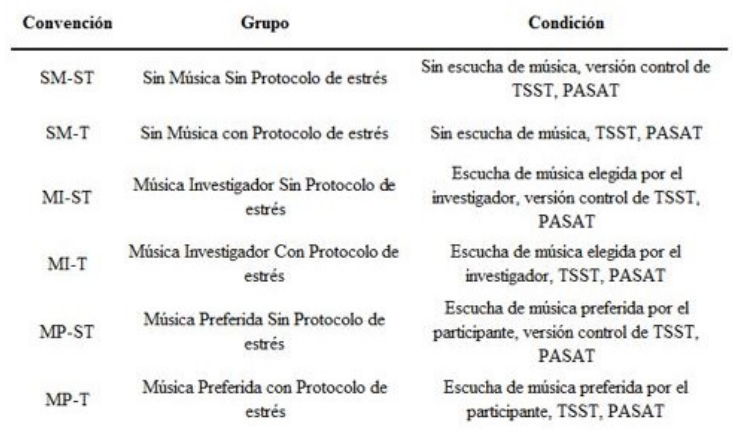

Fuente: elaboración propia.

\section{Instrumentos}

Los participantes que escucharon música usaban audífonos de balaca y un reproductor iPod para las piezas musicales. La música relajante seleccionada por los autores fue tomada del estudio de Nilsson (2009), caracterizada por tempo lento, ritmo suave, sin letra y por la inclusión de sonidos de la naturaleza. La música preferida por los participantes fueron canciones que les gustaría escuchar antes de dormir. El volumen de presentación fue modulado por los participantes. Las piezas musicales presentadas durante el procedimiento fueron seleccionadas aleatoriamente de dos listados previamente elaborados, según la condición, hasta completar 15 minutos de reproducción.

El protocolo de inducción de estrés y la versión correspondiente para los grupos sin estrés fue el mismo seguido por Tarazona et al. (2013). Para evaluar la percepción de estrés de la tarea se usó el Inventario de Ansiedad Rasgo-Estado IDARE (Spielberger \& Diaz-Guerrero, 1975), empleando la subescala de estado y haciendo una aplicación test-retest antes y después del protocolo TSST.

Para evaluar la atención sostenida y dividida se usó la prueba PASAT (Paced Auditory Serial 
Addition Test) siguiendo el mismo procedimiento de Tarazona et al. (2013).

A todos los participantes se les tomaron cuatro muestras de saliva usando dispositivos Salivette ${ }^{\circledR}$ y siguiendo el instructivo sugerido en el Test Inmunológico In Vitro para la determinación cuantitativa de cortisol en suero, plasma, orina, y saliva en humanos (Laboratorio Cobas S.L. 2009). Los análisis de las muestras fueron realizados en el Laboratorio Andreas Rothstein S.A. en la ciudad de Bogotá, utilizando procedimientos analíticos estandarizados para el uso clínico.

\section{Procedimiento}

Previo a la sesión experimental se solicitó a los participantes un listado con 10 canciones que les gustaría escuchar antes de dormir, y se les indicó que no debían consumir ningún alimento en la última hora previa a la hora citada, ni haber consumido bebidas alcohólicas, cigarrillo o sustancias psicoactivas 24 horas antes. Los estudiantes asistieron entre las 8:30 a.m. y las 12:00 p.m. teniendo en cuenta su jornada de estudio y que se han reportado mediciones comparables de respuestas fisiológicas ante situaciones de estrés social en la mañana y en la tarde (Kudielka, Schommer, Hellhammer, Kirschbaum, 2004). Al inicio de la sesión firmaban el consentimiento informado y diligenciaban una encuesta de condiciones generales para identificar algunos factores que pudieran afectar el desempeño de las tareas o la medición de cortisol en saliva. Al ingresar se les solicitaba hacer un enjuague bucal con agua y un lavado de manos para eliminar posibles contaminantes. Inmediatamente se les pedía tomar el algodón con sus manos, pasarlo por su boca y depositarlo en el tubo Salivette (muestra A). Luego debían esperar 15 minutos para la toma de la segunda muestra (muestra B), el pretest del IDARE, y la aplicación del protocolo TSST según el grupo (durante estos 15 minutos los participantes de los grupos con escucha de música usaron los audífonos y escucharon las piezas según la condición). Finalizado el TSST se tomaba la tercera muestra (muestra C), se aplicaba el post-test del IDARE y se les pedía que esperaran durante 15 minutos para la toma de la última muestra (muestra D). Durante este tiempo se aplicaba el PASAT. Al finalizar se les agradecía por su participación, se les daba un refrigerio y se les asignaba un puntaje adicional en una asignatura en curso.

\section{Consideraciones éticas}

El consentimiento informado y el procedimiento fueron avalados por el comité de ética de la Dirección General de Investigaciones de la Corporación Universitaria Minuto de Dios-Sede Principal. Además se siguieron las normativas nacionales para la investigación en salud y el código deontológico del psicólogo: Resolución n. ${ }^{\circ} 008430$ de 1993 del Ministerio de Salud y La Ley 1090 de 2006. Respecto a las normas mencionadas se aclara lo siguiente: a) en el consentimiento se aseguraba la confidencialidad de la información, se mencionaba el objetivo general del estudio y se informaba que los resultados globales serían divulgados al terminar el estudio y b) los riesgos de la investigación eran mínimos ya que se siguieron protocolos ampliamente usados en laboratorios de psicología.

\section{Análisis estadísticos}

Para evaluar los efectos de la escucha de música sobre el desempeño de las tareas del TSST se realizaron ANOVAs de una vía comparando los grupos que presentaron la prueba en los siguientes indicadores: a) puntuación emitida por los jueces de la exposición oral, b) número de errores cometidos durante la resta y c) número máximo al que llegaron en la resta. Para evaluar los efectos de la escucha de música sobre la respuesta de estrés provocada por el TSST se realizó una ANOVA de dos vías de medidas repetidas para los factores grupo y muestra de saliva, y una ANOVA de dos vías de medidas repetidas para los factores grupo y puntuación IDARE (pre-test y pos-test). Para establecer 
William Ruiz Gallo, Óscar G. Rodríguez-Angarita, Mayerli A. Prado-Rivera, D. Carolina CárdenasPoveda.

si existía una correlación entre la respuesta fisiológica (niveles de cortisol en saliva) y la valoración subjetiva de estrés (IDARE) se hizo un análisis de correlación empleando el coeficiente de Pearson entre los niveles de cortisol de la muestra $\mathrm{C}$ y la puntuación del IDARE posttest. Para evaluar los efectos de la interacción entre la escucha de música y el estrés sobre el desempeño del PASAT se realizaron ANOVAs de dos vías con factores escucha de música y protocolo de estrés para el bloque de 3 segundos y 2 segundos comparando: a) respuestas correctas, b) no respuestas, c) respuestas incorrectas y d) respuestas demoradas. En todos los análisis pos hoc se utilizó el método de comparaciones múltiples Holm-Sidak.

Para identificar el porcentaje de varianza explicado por los factores música y estrés en los indicadores evaluados, se hicieron modelos de regresión lineal simple en los que se incluyeron otros factores que también pudieran contribuir a dicha variación. Los factores adicionales fueron: edad, sexo, estrato, número de horas dormidas la noche anterior a la sesión experimental, número de horas transcurridas desde levantarse hasta la toma de la muestra A, tratamiento médico actual, síntomas de gripe; realización de actividad física, consumo de bebidas alcohólicas, cafeína, energizantes, cigarrillo o algún medicamento consumido en las últimas veinticuatro horas; promedio académico acumulado y si pertenecían al programa CONEXIÓN de Psicología UNIMINUTO el cual realiza acompañamiento académico a estudiantes con bajo rendimiento. Se usó el paquete estadístico SIGMASTAT 3.5 y para todas las pruebas se tomó como mínimo un nivel de significancia de $p<0.05$.

\section{Resultados}

Efectos de la escucha de música sobre el desempeño de las tareas del TSST

Las comparaciones realizadas entre los grupos que presentaron el TSST mostraron que no existen diferencias significativas para la puntuación de la exposición oral emitida por los jueces $\left(\mathrm{F}_{(2)}=0.073, p=0.929\right)$, para el número de errores cometidos durante la resta $\left(\mathrm{F}_{(2)}=\right.$ $0.142, p=0.868)$, ni para el número máximo al que llegaron en la resta $\left(F_{(2)}=0.456, p\right.$ $=0.637)$. Según los modelos de regresión, los síntomas de gripe $(t=2.645 ; p=0.012)$ predicen parcialmente la calificación de la exposición oral $\left(R^{2}=0.159\right)$; el ejercicio realizado el día anterior $(t=2.093 ; p=0.044)$ y el sexo $(t=2.180$; $p=0.036)$ predicen parcialmente los errores cometidos en la resta $\left(R^{2}=0.234\right)$; y el sexo $(t$ $=3.030 ; p=0.005)$ y el promedio académico $(t=2.337 ; p=0.025)$ predicen parcialmente el número máximo alcanzado en la resta $\left(R^{2}=\right.$ 0.264).

Efectos de la escucha de música sobre el nivel de cortisol y la valoración subjetiva de estrés

En la figura 1A y 1B se observa la comparación de la concentración de cortisol en saliva entre los grupos, en las cuatro muestras tomadas (A, B, $\mathrm{C}$ y D). Se encontraron diferencias significativas para los factores grupo $\left(\mathrm{F}_{(5)}=3.585, p=0.006\right)$, muestras de cortisol ( $\left.\mathrm{F}_{(3)}=9.095, p<.001\right)$, y la interacción grupo $\mathrm{x}$ muestras de cortisol $\left(\mathrm{F}_{(15)}=4.110, p<0.001\right)$. Los análisis pos hoc indicaron que las diferencias entre grupos fueron significativas en las muestras $\mathrm{C}$ (inmediatamente después del estrés) y D (15 minutos después): el grupo sin música con estrés (SM-T) mostró niveles de cortisol significativamente mayores a los del grupo sin música y sin estrés (SM-ST) en las muestras $\mathrm{C}(t=3.257, p=0.001)$ y $\mathrm{D}(t=$ $5.858, p<0.001)$, indicando que el protocolo del TSST provocó un incremento significativo en los niveles de cortisol en saliva, los cuales se mantuvieron elevados 15 minutos después de finalizada la situación de estrés.

Los participantes que escucharon música relajante y fueron expuestos a estrés (MI-T) no mostraron diferencias significativas con el grupo música relajante sin estrés (MI-ST) en las muestras $\mathrm{C}(t=.386, p=0.700)$ ni $\mathrm{D}(t=.908$, $p=0.365)$, indicando que la escucha de música 
relajante antes del TSST previno incrementos significativos en el nivel de cortisol en saliva debidos al protocolo; además, el nivel en $\mathrm{C}$ no fue significativamente mayor que en $\mathrm{B}(t=1.105$, $p=0.271)$ indicando que el aumento de nivel estaba ralentizado.

Los participantes del grupo que escucharon su música preferida y que estuvieron expuestos al protocolo de estrés (MP-T) no mostraron diferencias significativas con los participantes que escucharon música preferida sin exposición al protocolo (MP-ST) en la muestra $\mathrm{C}(t=1.211$, $p=0.228)$ pero sí en la $\mathrm{D}(t=2.091, p=0.038)$, mostrando que la escucha de música preferida por ellos demoró el incremento en los niveles de cortisol debidos al protocolo. El grupo MP-T no mostró niveles de cortisol significativamente superiores al grupo MI-T en las muestras C $(t=1.993, p=0.056)$ ni $\mathrm{D}(t=.976, p$ $=0.331)$, indicando que no existen diferencias significativas en los niveles de cortisol debido al tipo de música escuchada después de que los participantes estuvieran expuestos al protocolo de estrés. Así mismo, la varianza explicada por los modelos de regresión indicó que sólo el protocolo de estrés es predictor de los cambios en la muestra $\mathrm{C}\left(t=2.659, p=0.010 ; R^{2}=\right.$ $0.129)$ y $\mathrm{D}\left(t=3.202, p<0.001 ; R^{2}=0.313\right)$. Finalmente se encontró que el nivel de cortisol de los participantes no fue significativamente diferente en la línea de base (muestra A) ni inmediatamente después de la escucha de música (muestra B) indicando que las diferencias se observan luego del protocolo de estrés.

En relación con otros factores evaluados, los modelos de regresión mostraron que el número de horas transcurridas desde levantarse hasta la toma de la muestra A $(t=2.950, p=0.004)$ y los síntomas de gripe $(t=2.062, p=0.043)$ contribuyen en la predicción de la muestra $\mathrm{A}\left(R^{2}\right.$ $=0.139$ ) y ninguna de las variables evaluadas contribuye en la predicción de la muestra B.

\section{Figura 1}

Promedio $(\mathrm{SEM} \pm)$ de niveles de cortisol en saliva en las muestras CortA, B, C y D en los grupos sin estrés $(A)$ y con estrés $(B), y$ puntuaciones IDARE pre y post para los mismos grupos (C y D).
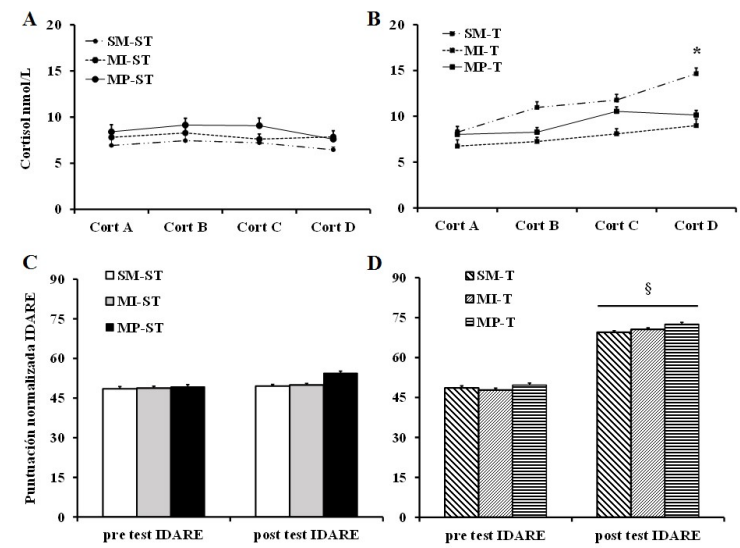

(*) indica diferencias significativas de los grupos MI-T y MP-T respecto del grupo $\mathrm{SM}-\mathrm{T}$ en CortD; $(\$)$ indica diferencias significativas con pre-test IDARE para cada grupo. Fuente: elaboración propia.

Al comparar la valoración subjetiva de estrés por la exposición al protocolo del TSST con la prueba IDARE aplicada antes (pre-test) y después (post-test) del TSST, se encontraron diferencias significativas para las variables grupo $\left(\mathrm{F}_{(5)}=7.751, p<0.001\right)$, puntuación IDARE $(\mathrm{F}$ (1) $=119.925, p<0.001)$, y la interacción grupo $x$ puntuación IDARE $\left(F_{(5)}=16.444, p<0.001\right)$. Los análisis pos hoc mostraron que los grupos que estuvieron expuestos al TSST tuvieron puntuaciones significativamente mayores en la valoración post-test comparada con la valoración pre-test (SM-T, $t=6.329, p<0.001$; MI-T $t=9.028, p<0.001 ;$ MP-T, $t=9.378, p<$ 0.001 ), lo que no ocurrió en los grupos que no estuvieron expuestos al TSST (SM-ST, $t=.351$, $p=0.726$; MI-ST $t=.441, p=0.661$; MP-ST, $t$ $=1.968, p=0.053)$, indicando que únicamente los grupos expuestos al protocolo valoraron la situación como estresante. No se encontraron diferencias entre ningún grupo en la valoración pre-test, mostrando que la apreciación subjetiva después de la música y antes del protocolo de estrés fue semejante entre los grupos. Tampoco 
William Ruiz Gallo, Óscar G. Rodríguez-Angarita, Mayerli A. Prado-Rivera, D. Carolina CárdenasPoveda.

se encontraron diferencias entre los grupos expuestos a estrés en la puntuación post-test (MP-T vs. SM-T, $t=.807, p=0.421$; MP-T vs. MI-T, $t=.614, p=0.540$; MI-T vs. SM-T, $t$ $=.245, p=0.807$ ), indicando que la valoración subjetiva ante el TSST fue independiente del tipo de música escuchada.

Los modelos de regresión indicaron que ninguno de los factores evaluados contribuye a la predicción de la puntuación pre-test del IDARE; y que las variables sexo $(t=2.100, p=0.039)$ y estrés $(t=9.776, p<0.001)$ explican un porcentaje de la varianza de la puntuación posttest del IDARE $\left(R^{2}=0.587\right)$, consistente con lo hallado en el análisis de varianza.

Al correlacionar los niveles de cortisol entre la muestra $\mathrm{C}$ y los valores de la prueba IDARE post-test para cada grupo, se encontró que no existen asociaciones moderadas o fuertes entre los resultados del indicador fisiológico y la valoración subjetiva de estrés.

Efectos de la escucha de música y el estrés sobre el desempeño del PASAT

No se encontraron diferencias significativas para los factores escucha de música, protocolo, ni su interacción en ninguna de las medidas en ninguno de los bloques. Los modelos de regresión múltiple para las medidas del bloque 3 segundos arrojaron que las respuestas correctas $\left(R^{2}=0.155\right)$ se pueden predecir a partir de las variables promedio académico $(t=2.343, p=$ $0.022)$ y síntomas de gripe $(t=-2,781, p=$ $0.007)$, al igual que las respuestas incorrectas $\left(R^{2}=0.126\right.$; promedio académico: $t=-2.407$, $p=0.019$; Síntomas de gripe: $t=2.124, p=$ 0.019 ); mientras que ninguna de las variables evaluadas pueden predecir las no respuestas y respuestas tardías. Los modelos aplicados para las medidas del bloque 2 segundos arrojaron que las respuestas correctas $\left(R^{2}=0.108\right)$ se pueden predecir a partir de las variables síntomas de gripe $(t=-2.225, p=0.029)$ y horas transcurridas entre levantarse y la primera muestra $(t=2.190$, $p=0.032) ;$ Las respuestas tardías $\left(R^{2}=0.145\right)$ se pueden predecir a partir del ejercicio físico $(t$ $=3.539, p<0.001)$; y ninguna de las variables evaluadas pueden predecir las no respuestas y respuestas incorrectas.

\section{Discusión}

La resolución de problemas contribuye en el éxito académico y laboral, por lo cual es útil identificar o desarrollar herramientas que la faciliten. Los resultados del primer objetivo en este estudio mostraron que la escucha de música previa a la resolución de una tarea aritmética y una exposición oral no tuvo efectos facilitadores o deletéreos. Jaušovec et al. (2006) evidenciaron al igual que Rauscher et al. (1993), que la escucha de música parece facilitar tareas espaciales, pero algunos estudios muestran un desempeño similar entre grupos con y sin escucha de música en la resolución de este tipo de tareas (Schellenberg \& Hallam, 2005) y de tareas matemáticas (Jaušovec, Norbert, \& Katarina, 2003). Considerando que Schellenberg et al. (2013) proponen que el efecto de la música escuchada sobre varias tareas depende más del estado emocional que ésta provoca y del tipo de tarea que de la música per se, el probable estado emocional provocado por la música elegida por los investigadores o preferida por los participantes en este estudio, no afectó tareas aritméticas o la presentación de un discurso. Debe hacerse un estudio posterior que permita identificar el tipo de estados emocionales causados por la escucha de música (tanto preferida como elegida por otros) y su interacción con el desarrollo de este tipo de tareas, en un diseño que permita disociar los procesos cognitivos que implica una tarea tan compleja como una exposición oral.

Los modelos de regresión realizados mostraron que la variación en los indicadores de desempeño del TSST puede explicarse por variables asociadas a la salud de los participantes como practicar ejercicio físico. Aunque el porcentaje de varianza explicada en este caso fue pequeño, lo anterior hace necesario valorar de manera más controlada estos aspectos teniendo en cuenta que otros estudios reportan los problemas de 
salud como variables que afectan el rendimiento escolar (Needham, Crosnoe, \& Muller, 2004).

El sexo y promedio académico también aparecieron en los modelos. En relación con la primera variable, algunos estudios muestran que los hombres tienen mejor desempeño que las mujeres en tareas matemáticas (Guiso, Monte, Sapienza, \& Zingales, 2008), aunque cualquier conclusión al respecto debe hacerse con cuidado pues esta diferencia desaparece en culturas donde hombres y mujeres reciben educación semejante (León, Federico, \& Consultores, 2013). Con respecto al promedio académico, Edel (2003) encontró que el puntaje de matemáticas en un examen de admisión para la educación superior correlacionó con el promedio de calificaciones de primer año de preparatoria, sugiriendo que funciones cognitivas que se requieren para este tipo de tareas son capacidades estables sobre las que intervenciones tan rápidas como la escucha de música por un periodo muy corto no tienen influencia inmediata.

La validación ecológica de protocolos de inducción de estrés en humanos para estudiar su relación con diversas variables, es decir protocolos que se acerquen a experiencias reales y cotidianas de las personas, implica procedimientos controlados. En este estudio al igual que en otros (Kirschbaum et al., 1993; Schoofs \& Wolf, 2011; Tarazona et al., 2013) se encontró que los niveles de cortisol en saliva de los participantes expuestos al protocolo del TSST fueron significativamente superiores inmediatamente y 15 minutos después comparados con los grupos expuestos a la versión de control, y este incremento también se observó en la valoración subjetiva de estrés evaluada con la prueba IDARE. El grupo que escuchó música seleccionada por los investigadores mostró un incremento más lento de concentración de cortisol al igual que el grupo que escuchó su música preferida, en especial entre las últimas dos muestras. Teniendo en cuenta que la escucha de música puede provocar respuestas emocionales (Krumhansl, 1997; Rickard, 2004; Peretz, 2010; Schellenberg et al., 2013; Juslin \& Sloboda, 2013), la ralentización del incremento del nivel de cortisol que se encontró en este estudio, podría reflejar que la escucha de música disminuye la reactividad emocional asociada al eje HPA provocando que al estar expuestos a una situación de estrés, la respuesta sea menos intensa. Así mismo, este hallazgo muestra que la escucha de música previa a una tarea estresante modula el nivel de liberación de cortisol de manera similar a cuando la música se presenta después de este tipo de tareas (Khalfa et al., 2003), y que este efecto es independiente de la elección de la música (preferida o elegida por el investigador), lo que puede indicar que el estado emocional provocado por el estímulo musical es más importante que sus características intrínsecas (tempo, ritmo, etc.) en la modulación de las respuestas fisiológicas de estrés. Esto es además consistente con el estudio de le Roux, Bouic, \& Bester (2007) en el que la música seleccionada por el investigador redujo niveles de cortisol en pacientes con problemas pulmonares a pesar de que la pieza musical tuviera características activadoras. De esta manera es necesario considerar factores individuales, situacionales y de interpretación del estímulo musical para evaluar el alcance de la escucha de música en la modulación de respuestas de estrés.

Por otro lado, la valoración subjetiva de estrés no fue modulada por la escucha de música, ni se encontró correlación entre ella y los niveles de cortisol evaluados después del TSST, lo que es coherente con algunos estudios en los que la respuesta psicológica de estrés y medidas fisiológicas no convergen (Campbell \& Ehlert, 2012; Cohen et al., 2000; Hjortskov, Nis, Garde, Palle, \& Hansen, 2004). Algunos autores sugieren que esto puede ocurrir debido a que existen dinámicas temporales diferentes de los sistemas que regulan las respuestas psicológicas y fisiológicas de estrés, siendo la percepción subjetiva susceptible de cambios rápidos y altamente sensible, mientras que las respuestas endocrinas son más lentas (Hellhammer, Juliane, \& Melanie, 2012; Schlotz et al., 2008). Lo anterior una vez más muestra la necesidad de medición de las respuestas emocionales en varios momentos antes, durante y después de la realización de este tipo de tareas. 
William Ruiz Gallo, Óscar G. Rodríguez-Angarita, Mayerli A. Prado-Rivera, D. Carolina CárdenasPoveda.

Una variable diferente que apareció en los modelos de regresión fue el número de horas transcurridas desde que los estudiantes se levantaron hasta que se tomó la primera muestra de saliva. La liberación de cortisol no solo depende de la respuesta rápida del eje HPA ante la aparición de un estímulo concreto como un estresor, sino también de ritmos circadianos que inducen secreciones pulsátiles. Las horas de mayor fluctuación en humanos ocurren entre las primeras horas de la madrugada cuando los niveles de cortisol son bajos y cerca de iniciar el periodo activo cuando los niveles son los más altos del ciclo (Collomp et al., 2016; Mazzoccoli et al., 2011), pero este patrón natural fluctúa a su vez a partir del número de horas de sueño, del momento en el que las personas se duermen y se despiertan (Abell, Shipley, Ferrie, Kivimäki, \& Kumari, 2016), de la edad, el género, y la genética, entre otros (Kalafatakis, Russell, Zarros, \& Lightman, 2016). Todas las muestras de este estudio se tomaron en horas de la mañana, y si bien los niveles de cortisol en saliva fueron sensibles al estrés inducido, identificar diferentes patrones de fluctuación asociados al sueño plantea la necesidad de estudiar esta relación, ya que alteraciones constantes en el funcionamiento basal del cortisol pueden desregular el mecanismo de retroalimentación del eje HPA afectando a largo plazo la reactividad al estrés e incrementar el riesgo de diversas psicopatologías (Abell et al., 2016). Asimismo, teniendo en cuenta que el $34,21 \%$ de los participantes reportaron síntomas de gripe y esta variable también se encontró relacionada con la primera medición de concentración de cortisol, es importante describir con mayor detalle las condiciones de salud de los estudiantes universitarios ya que las infecciones están relacionadas con la acción de inmunosupresores que tienen efectos sobre funciones cognitivas tal como lo reportan algunos estudios en los que se observó una mala ejecución en una tarea de aprendizaje verbal desde el primer día de administración de prednisona en sujetos sanos (Brown, Beard, Frol, \& Rush, 2006) o en sujetos en tratamiento con prednisona o betametasona por dermatitis o enfermedades reumáticas pero mejoras en tareas de velocidad psicomotora, atención y memoria de trabajo (Hájek, Kopecek, Preiss, Alda, \& Höschl, 2006), mostrando que los efectos de los inmunosupresores exógenos sobre funciones cognitivas son evidentes y variados.

Para el tercer objetivo de este estudio los resultados mostraron que la escucha de música sumada con la presentación de un protocolo de estrés social no tuvo efectos facilitadores o deletéreos en el desempeño en la tarea PASAT en ninguna de sus dos modalidades, en ningún indicador. Previamente se ha descrito que el estrés agudo no afecta de manera significativa la ejecución en tareas de atención (Hoffman \& Al'Absi, 2004; Tarazona et al., 2013), y al parecer los cambios fisiológicos inducidos por la escucha de música no son suficientes para afectar el desempeño del PASAT. Se sabe que los efectos de los glucocorticoides liberados por situaciones de estrés siguen un patrón en U-invertida, significando que niveles muy bajos o muy altos afectan tareas como la memoria de trabajo (Oei, Everaerd, Elzinga, van Well, \& Bermond, 2006) o la memoria verbal (Kirschbaum, Wolf, May, Wippich, \& Hellhammer, 1996), pero niveles medios no tienen efectos o pueden ser facilitadores (Wolf, Schommer, Hellhammer, McEwen, \& Kirschbaum, 2001). De esta manera, es posible que el aumento en la concentración de cortisol hallado en este estudio no alcanza a provocar niveles muy elevados que afectaran el desempeño de la tarea y que la ralentización del aumento provocada por la escucha de música evitará que llegara a tales niveles, sugiriendo un efecto regulador sobre la concentración de cortisol. Aunque los modelos de regresión también evidenciaron porcentajes pequeños de explicación de la varianza en los indicadores del desempeño de la prueba PASAT, cabe destacar que el promedio académico, los síntomas de gripe, el ejercicio físico, y las horas transcurridas hasta la primera muestra de cortisol surgen nuevamente relacionadas con algunos indicadores de la tarea, evidenciando que las condiciones de salud y el nivel académico de las personas pueden ser predictores del desempeño en actividades académicas y laborales que exijan 
atención y memoria de trabajo, incidiendo directamente sobre su calidad.

En conclusión, este estudio mostró que escuchar música tiene un efecto sobre la respuesta fisiológica de estrés posiblemente por la inducción de reacciones emocionales que necesitan ser identificadas, indicando que la música puede jugar un papel importante en el diseño y ejecución de estrategias para la regulación emocional ante situaciones de estrés, teniendo en cuenta que su uso ha venido creciendo como parte de intervenciones terapéuticas, pero no se tiene claridad sobre los mecanismos subyacentes que ésta regula y que permitan explicar su efectividad.

\section{Agradecimiento}

Este estudio fue financiado por recursos internos de la Corporación Universitaria Minuto de Dios Sede Principal por medio de la IV Convocatoria para el Fortalecimiento de la Investigación Uniminuto, (código: C113-55) proyecto: "La música relajante como factor modulador de respuestas de estrés provocadas por la realización de tareas académicas: estudio experimental en estudiantes de psicología Uniminuto".

\section{Referencias}

Abell, J. G., Shipley, M. J., Ferrie, J. E., Kivimäki, M., \& Kumari, M. (2016). Recurrent short sleep, chronic insomnia symptoms and salivary cortisol: A 10 year follow-up in the Whitehall II study. Psychoneuroendocrinology, 68, 91-99. http:// doi.org/10.1016/j.psyneuen.2016.02.021

Allen, A. P., Kennedy, P. J., Cryan, J. F., Dinan, T. G., \& Clarke, G. (2014). Biological and psychological markers of stress in humans: focus on the Trier Social Stress Test. Neuroscience and Biobehavioral Reviews, 38, 94-124. http://doi.org/10.1016/j.neubiorev .2013 .11 .005

Ashby, F. G., Isen, A. M., \& Turken, A. U. (1999). A neuropsychological theory of positive affect and its influence on cognition. Psychological Review, 106

Brown, E. S., Beard, L., Frol, A. B., \& Rush, A. J. (2006). Effect of two prednisone exposures on mood and declarative memory. Neurobiology of Learning and Memory, 86(1), 28-34. http://doi.org/10.10 16/j.nlm.2005.12.009

Buchanan, T. W., David, D., Mowrer, S. M., Sollers, J. J., Thayer, J. F., Clemens, K., \& Daniel, T. (2010). Medial prefrontal cortex damage affects physiological and psychological stress responses differently in men and women. Psychoneuroendocrinology, 35 (1), 56-66. http://doi.org/10.1016/j.psyn euen.2009.09.006

Campbell, J., \& Ehlert, U. (2012). Acute psychosocial stress: does the emotional stress response correspond with physiological responses? Psychoneuroendocrinology, 37(8), 11111134. http://doi.org/10.1016/j.psyneuen.20 11.12 .010

Chanda, M. L., \& Levitin, D. J. (2013). The neurochemistry of music. Trends in Cognitive Sciences, 17(4), 179-193. http://doi.org/10. 1016/j.tics.2013.02.007

Cohen, S., Hamrick, N., Rodríguez, M. S., Feldman, P. J., Rabin, B. S., \& Manuck, S. B. (2000). The stability of and intercorrelations among cardiovascular, immune, endocrine, and psychological reactivity. Annals of Behavioral Medicine: A Publication of the Society of Behavioral Medicine, 22(3), 171-179.

Collomp, K., Baillot, A., Forget, H., Coquerel, A., Rieth, N., \& Vibarel-Rebot, N. (2016). Altered diurnal pattern of steroid hormones in relation to various behaviors, external factors and pathologies: A review. Physiology EF Behavior, 164(Pt A), 6885. http://doi.org/10.1016/j.physbeh.2016. 05.039

de Quervain, D. J.-F., Henke, K., Aerni, A., Treyer, V., McGaugh, J. L., Berthold, T., ... Hock, C. (2003). Glucocorticoid-induced impairment of declarative memory retrieval is associated with reduced blood flow in the 
William Ruiz Gallo, Óscar G. Rodríguez-Angarita, Mayerli A. Prado-Rivera, D. Carolina CárdenasPoveda.

medial temporal lobe. The European Journal of Neuroscience, 17(6), 1296-1302.

Duncko, R., Johnson, L., Merikangas, K., \& Grillon, C. (2009). Working memory performance after acute exposure to the cold pressor stress in healthy volunteers. Neurobiology of Learning and Memory, 91 (4), 377-381.

Ebneshahidi, A., \& Mohseni, M. (2008). The effect of patient-selected music on early postoperative pain, anxiety, and hemodynamic profile in cesarean section surgery. Journal of Alternative and Complementary Medicine, 14(7), 827-831. h ttp://doi.org/10.1089/acm.2007.0752

Edel, R. (2003). Factores asociados al rendimiento académico. Revista Iberoamericana de Educación. Organización de Estados Iberoamericanos para la Educación, la Ciencia y la Cultura. En red. Retrieved from: http://www.campus-oei.o rg/revista/frame_participar.htm . Sección: Investigación, 20 de Septiembre de 2003.

Guiso, L., Monte, F., Sapienza, P., \& Zingales, L. (2008). DIVERSITY: Culture, Gender, and Math. Science, 320(5880), 1164-1165. http ://doi.org/10.1126/science.1154094

Hájek, T., Kopecek, M., Preiss, M., Alda, M., \& Höschl, C. (2006). Prospective study of hippocampal volume and function in human subjects treated with corticosteroids. European Psychiatry: The Journal of the Association of European Psychiatrists, 21(2), 123-128. http://doi.org /10.1016/j.eurpsy.2005.01.005

Hellhammer, J., Juliane, H., \& Melanie, S. (2012). The physiological response to Trier Social Stress Test relates to subjective measures of stress during but not before or after the test. Psychoneuroendocrinology, 37(1), 119-124. http://doi.org/10.1016/j.ps yneuen.2011.05.012

Het, S., Ramlow, G., \& Wolf, O. T. (2005). A meta-analytic review of the effects of acute cortisol administration on human memory. Psychoneuroendocrinology, 30(8), 771-784. http://doi.org/10.1016/j.psyneue n.2005.03.005
Hjortskov, N., Nis, H., Garde, A. H., Palle, Ø., \& Hansen, А. M. (2004). Evaluation of salivary cortisol as a biomarker of selfreported mental stress in field studies. Stress and Health: Journal of the International Society for the Investigation of Stress, 20(2), 91-98. http://doi.org/10.1002/smi.1000

Hoffman, R., \& Al'Absi, M. (2004). The effect of acute stress on subsequent neuropsychological test performance (2003). Archives of Clinical Neuropsychology: The Official Journal of the National Academy of Neuropsychologists, 19(4), 497-506. http: //doi.org/10.1016/j.acn.2003.07.005

Isen, A. M. (2009). A Role for Neuropsychology in Understanding the Facilitating Influence of Positive Affect on Social Behavior and Cognitive Processes. http://doi.org/10.1093 /oxfordhb/9780195187243.013.0048

Isen, A. M., \& Labroo, A. A. (2003). Some Ways in Which Positive Affect Facilitates Decision Making and Judgment. In Schneider, S. L. \& Shanteau, J. (Eds.), Emerging Perspectives on Judgment and Decision Research (pp. 365-393). New York, NY: Cambridge University Press. http ://doi.org/10.1017/cbo9780511609978.013

Jaušovec, N., Jaušovec, K., \& Gerlic, I. (2006). The influence of Mozart's music on brain activity in the process of learning. Clinical Neurophysiology: Official Journal of the International Federation of Clinical Neurophysiology, 117(12), 2703-2714. http: //doi.org/10.1016/j.clinph.2006.08.010

Jaušovec, N., Norbert, J., \& Katarina, H. (2003). The "Mozart Effect": An Electroencephalographic Analysis Employing the Methods of Induced Event-Related Desynchronization/ Synchronization and Event-Related Coherence. Brain Topography, 16(2), 73 84. http://doi.org/10.1023/b:brat.00000063 31.10425.4b

Jiang, J., Jun, J., Linshu, Z., Daphne, R., \& Cunmei, J. (2013). The effects of sedative and stimulative music on stress reduction depend on music preference. The Arts in 
Psychotherapy, 40(2), 201-205. http://doi.o rg/10.1016/j.aip.2013.02.002

Johnson, A. C. (2015). Developmental pathways to attention-deficit/hyperactivity disorder and disruptive behavior disorders: Investigating the impact of the stress response on executive functioning. Clinical Psychology Review, 36, 1-12. http://doi.org/ 10.1016/j.cpr.2014.12.001

Juslin, P. N. \& Sloboda, J. A. (2013). Music and Cognitive Abilities. In Deutsch, D. The Psychology of Music (pp. 583-645). http://dx.doi.org/10.1016/B9780-12-381460-9.00015-8

Kalafatakis, K., Russell, G. M., Zarros, A., \& Lightman, S. L. (2016). Temporal control of glucocorticoid neurodynamics and its relevance for brain homeostasis, neuropathology and glucocorticoidbased therapeutics. Neuroscience and Biobehavioral Reviews, 61, 12-25. http://doi .org/10.1016/j.neubiorev.2015.11.009

Khalfa, S., Bella, S. D., Roy, M., Peretz, I., \& Lupien, S. J. (2003). Effects of relaxing music on salivary cortisol level after psychological stress. Annals of the New York Academy of Sciences, 999, 374-376.

Kirschbaum, C., Pirke, K. M., \& Hellhammer, D. H. (1993). The "Trier Social Stress Test"-. a tool for investigating psychobiological stress responses in a laboratory setting. Neuropsychobiology, 28(1-2), 76-81. http:// doi.org/119004

Kirschbaum, C., Wolf, O. T., May, M., Wippich, W., \& Hellhammer, D. H. (1996). Stress- and treatment-induced elevations of cortisol levels associated with impaired declarative memory in healthy adults. Life Sciences, 58(17), 1475-1483.

Knight, W. E., \& Rickard, N. S. (2001). Relaxing music prevents stress-induced increases in subjective anxiety, systolic blood pressure, and heart rate in healthy males and females. Journal of Music Therapy, 38(4), 254-272.

Krumhansl, Carol L. (1997). An exploratory study of musical emotions and psychophysiology. Canadian Journal of Experimental Psychology/Revue canadienne de psychologie expérimentale, 51(4), 336-353. http://dx.doi.org/10.1037/1196-1 961.51.4.336

Kudielka, B.M., Schommer, N.C., Hellhammer, D.H., Kirschbaum, C. (2004). Acute HPA axis responses, heart rate, and mood changes to psychosocial stress (TSST) in humans at different times of day. Psychoneuroendocrinology, 29, 983-992. htt p://doi:10.1016/j.psyneuen.2003.08.009

Kudielka B.M., Hellhammer D.H. \& Kirschbaum C. (2007). Ten years of research with the Trier Social Stress Test - revisited. In: E. Harmon-Jones \& P. Winkielman (Eds.), Social neuroscience: integrating biological and psychological explanations of social behavior, chapter 4, pp. 56-83. New York: The Guilford Press.

Lai, V., Viet, L., Chalermsiri, T., Tadao, M., Yoshiyuki, H., Mehrnoosh, M., ... Masaharu, K. (2014). A normal intensity level of psycho-physiological stress can benefit working memory performance at high load. International Journal of Industrial Ergonomics, 44(3), 362-367. http://doi.org/ 10.1016/j.ergon.2013.11.015

Lataster, J., Collip, D., Ceccarini, J., Haas, D., Booij, L., van Os, J., ... MyinGermeys, I. (2011). Psychosocial stress is associated with in vivo dopamine release in human ventromedial prefrontal cortex: a positron emission tomography study using [18F] fallypride. NeuroImage, 58(4), 10811089. http://doi.org/10.1016/j.neuroimage. 2011.07.030

Leardi, S., Pietroletti, R., Angeloni, G., Necozione, S., Ranalletta, G., \& Del Gusto, B. (2007). Randomized clinical trial examining the effect of music therapy in stress response to day surgery. The British Journal of Surgery, 94(8), 943-947. http://d oi.org/10.1002/bjs.5914

León, F., Federico, L., \& Consultores, L. \&. B. (2013). Diferencias de sexo en matemática y comprensión lectora según poder femenino, urbanización y habilidad. Propósitos Y Representaciones, 1(1), 11-37. h ttp://doi.org/10.20511/pyr2013.v1n1.1 
William Ruiz Gallo, Óscar G. Rodríguez-Angarita, Mayerli A. Prado-Rivera, D. Carolina Cárdenas-

Poveda.

le Roux, F. H., Bouic, P. J. D., \& Bester, M. M. (2007). The Effect of Bach's Magnificat on Emotions, Immune, and Endocrine Parameters During Physiotherapy Treatment of Patients with Infectious Lung Conditions. Journal of Music Therapy, 44(2), 156-168. http://doi. org $/ 10.1093 / \mathrm{jmt} / 44.2 .156$

Lupien, S. J., Maheu, F., Tu, M., Fiocco, A., \& Schramek, T. E. (2007). The effects of stress and stress hormones on human cognition: Implications for the field of brain and cognition. Brain and Cognition, 65(3), 209-237. http://doi.org/10.1016/j.bandc.20 07.02.007

Mason, J. W. (1968). A review of psychoendocrine research on the sympathetic-adrenal medullary system. Psychosomatic Medicine, 30(5), Suppl:63153. Retrieved from http://www.ncbi.nlm.ni h.gov/pubmed/4974233

Mazzoccoli, G., Gianluigi, M., Sothern, R. B., Valerio, P., Ada, P., Muscarella, L. A., ... Francesco, G. (2011). Chronobiologic study of neuro-endocrine axis hormone sequence signalling in healthy men. Biomedicine $\mathcal{E}$ Aging Pathology, 1(3), 129-137. http://doi.o rg/10.1016/j.biomag.2011.06.008

McEwen, B. S. (2007). Physiology and Neurobiology of Stress and Adaptation: Central Role of the Brain. Physiological Reviews, 87(3), 873-904. http://doi.org/10. 1152/physrev.00041.2006

Mizoguchi, K., Yuzurihara, M., Ishige, A., Sasaki, H., Chui, D. H., \& Tabira, T. (2000). Chronic stress induces impairment of spatial working memory because of prefrontal dopaminergic dysfunction. The Journal of Neuroscience: The Official Journal of the Society for Neuroscience, 20(4), 15681574.

Needham, B. L., Crosnoe, R., \& Muller, C. (2004). Academic Failure in Secondary School: The Inter-Related Role of Health Problems and Educational Context. Social Problems, 51(4), 569-586.

Nilsson, U. (2009). The effect of music intervention in stress response to cardiac surgery in a randomized clinical trial. Heart Lung 38, 201-207.

Oei, N. Y. L., Everaerd, W. T. A. M., Elzinga, B. M., van Well, S., \& Bermond, B. (2006). Psychosocial stress impairs working memory at high loads: an association with cortisol levels and memory retrieval. Stress, 9(3), 133-141. http://doi.org/10.1080/10253890 600965773

Ortega, L. A., Prado-Rivera, M. A., CárdenasPoveda, D.C, McLinden, K. A., Glueck, A. C., Gutierrez, G.A, Lamprea, M., \& Papini, M. R. (2013). Tests of the aversive summation hypothesis in rats: Effects of restraint stress on consummatory successive negative contrast and extinction in the Barnes maze. Learning and Motivation, 44(3), 159-173.

Peretz I. (2010). Towards a neurobiology of musical emotions. In Juslin P. N., Sloboda J. A. (Eds.). Handbook of Music and Emotion: Theory, Research, Applications. Oxford: Oxford University Press, 99-126. DOI: 10.1093/ acprof:oso/9780199230143.001.0001

Porcelli, A. J., Cruz, D., Wenberg, K., Patterson, M. D., Biswal, B. B., \& Rypma, B. (2008). The effects of acute stress on human prefrontal working memory systems. Physiology $\mathcal{E}$ Behavior, 95 (3), 282-289. http ://doi.org/10.1016/j.physbeh.2008.04.027

Rauscher, F. H., Shaw, G. L., \& Ky, C. N. (1993). Music and spatial task performance. Nature, 365(6447), 611-611. http://doi.org $/ 10.1038 / 365611 \mathrm{aO}$

Rickard, N. S. (2004). Intense emotional responses to music: a test of the physiological arousal hypothesis. Psychology of Music, 32, 371-388. http://doi.org/10.117 7/0305735604046096

Sandi, C. (2011). Glucocorticoids act on glutamatergic pathways to affect memory processes. Trends in Neurosciences, 34(4), 165-176. http://doi.org/10.1016/j.tins.2011 .01 .006

Schellenberg, E. G., Glenn Schellenberg, E., \& Weiss, M. W. (2013). Music and Cognitive Abilities. In The Psychology of Music (pp. 
499-550). http://doi.org/10.1016/b978-0-1 2-381460-9.00012-2

Schellenberg, E. G., \& Hallam, S. (2005). Music listening and cognitive abilities in 10- and 11-year-olds: the blur effect. Annals of the New York Academy of Sciences, 1060, 202209. http://doi.org/10.1196/annals.1360.01 3

Schlotz, W., Wolff, S., Robert, K., Irmgard, L., Sonja, E., Alexander, J., \& Stefan, W. (2008). Covariance Between Psychological and Endocrine Responses to Pharmacological Challenge and Psychosocial Stress: A Question of Timing. Psychosomatic Medicine, 70(7), 787-796. http://doi.org/10.1097/psy.0b013 e3181810658

Schoofs, D., Pabst, S., Brand, M., \& Wolf, O. T. (2013). Working memory is differentially affected by stress in men and women. Behavioural Brain Research, 241, 144-153. h ttp://doi.org/10.1016/j.bbr.2012.12.004

Schoofs, D., \& Wolf, O. T. (2011). Are salivary gonadal steroid concentrations influenced by acute psychosocial stress? A study using the Trier Social Stress Test (TSST). International Journal of Psychophysiology: Official Journal of the International Organization of Psychophysiology, 80(1), 3643. http://doi.org/10.1016/j.ijpsycho.2011. 01.008

Schwabe, L., Lars, S., Marian, J., Benno, R., Wolf, O. T., \& Oitzl, M. S. (2012). Stress effects on memory: An update and integration. Neuroscience and Biobehavioral Reviews, 36(7), 1740-1749. http://doi.org/1 0.1016/j.neubiorev.2011.07.002

Spielberger, Ch., \& Díaz-Guerrero, R. (1975). IDARE Inventario de ansiedad: rasgo - estado. Manual Moderno.

Tarazona, O., Cerón, J., \& Lamprea, M. (2013). Efecto de la Exposición a un Protocolo de Estrés Social Agudo sobre los Niveles Sistémicos de Cortisol y la Ejecución de una Tarea de Atención Sostenida y Dividida. Revista Colombiana de Psicología, 22(2), 347-360.
Test inmunológico in vitro para la determinación cuantitativa de cortisol en suero, plasma, orina y saliva humanos (Ref.11875116 122). (2009). Madrid: Laboratorio Cobas S.L.

Wolf, O. T., Schommer, N. C., Hellhammer, D. H., McEwen, B. S., \& Kirschbaum, C. (2001). The relationship between stress induced cortisol levels and memory differs between men and women. Psychoneuroendocrinology, 26(7), 711-720.

Zwissler, B., Bastian, Z., Susanne, K., Harald, E., Manfred, S., \& Johanna, K. (2011). Acute psycho-social stress does not disrupt item-method directed forgetting, emotional stimulus content does. Neurobiology of Learning and Memory, 95 (3), 346-354. http ://doi.org/10.1016/j.nlm.2011.01.007

\section{Notas}

* Artículo de investigación. 